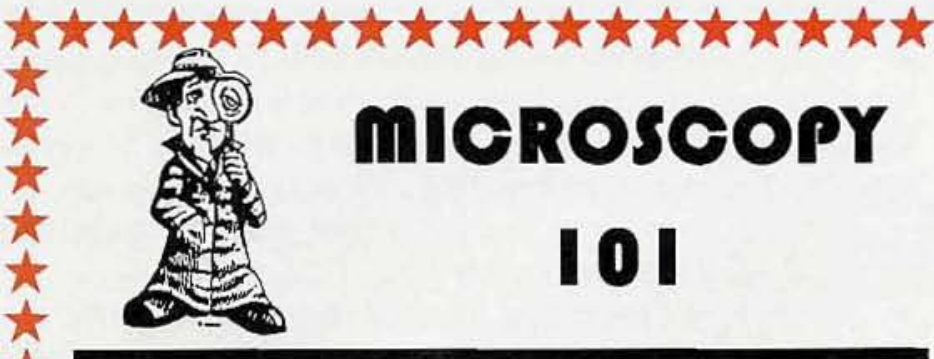

We appreciate the response to this publication feature - and welcome all contributions. Contributions may be sent to Phil Oshel, our Technical Editor at:

Mr. Phil Oshel

Station A

PO Box 5037

Champaign iL 61825-5037

or by eMail: poshel@hotmail.com

Please note new eMail address

\section{A Protocol for Transmission Electron Microscopy of Secondary Vascular Tissues of Hardwood Trees:}

TEM of the secondary vascular tissues - secondary $x$ lem $_{1}$ cambium, secondary xylem - of hardwood trees is difficult. However, if the sampling guidelines in the article "Big is Beautiful: Science (and Art) in Wood Microscopy" (Microscopy Today, November 1997, Issue 97-9, pg. 12-14) are followed, the procedure below should give good results.

1) Fix in a solution of $3.7 \%$ freshly-made p formaldehyde and $2.5 \%$ glutaraldehyde in $25 \mathrm{mM}$ Piperazine-N, $\mathrm{N}^{\prime}$-bis[2-ethanesulphonic acid], $\mathrm{pH}$ 6.9. (PIPES buffer) for ca. 3 hours

2) Wash ca. $3 \times 5$ minutes in PIPES buffer

3) Trim to final block size in PIPES buffer

4) Osmicate in $1 \% \mathrm{OsO}_{4}$ for ca. 1.5 hours

5) Wash ca. $2 \times 5$ minutes in water, store in water overnight

6) Dehydrate in ethanol, 30 minutes each at $30,50,70,80,90 \%$ and $3 x$

30 minutes in $100 \%$. Follow with $2 \times 30$ minutes propylene oxide. To final propylene oxide step, add equal volume of Spurr's resin (hard grade) and leave overnight

7) Replace half volume with pure Spurr's resin, leave for ca. 9 hours

8) Replace all volume with pure Spurr's resin and leave overnight (ca. 15-20 hours)

9) Replace all volume with pure Spurr's resin and leave for ca. 5.5 hours 10) Replace all volume with pure Spur's resin and leave for ca. 4.5 hours NB: All above steps $1-10$ at room temperature on rotator in stoppered glass vials.

11) Embed in polythene capsules overnight at $65^{\circ} \mathrm{C}$

12) Cut sections at ca. $60-80 \mathrm{~nm}$, collect on uncoated grids

13) Post-stain with saturated uranyl acetate, and Fahmy's lead citrate, 30 minutes each (needed for adequate contrast)

Nigel Chaffey.

Swedish University of Agricultural Sciences. Umeá, Sweden

\section{Chemical Etching of Silicon:}

A question was asked on the microscopy listserver by Kirill $E$. Prikhodko about chemical etching of silicon for TEM. Several readers of the server sent Dr. Prikhodko suggestions, and while some used the same chemicals, none were identical to the answer posted by Dr. Prikhodko in his summary of responses, and we are publishing just his final post as made to the listserver and passed on to us.
First of all, why use chemical etching?

1) Various jet techniques require special equipment that allow the use of $\mathrm{HF}$ base solutes, which not everyone has.

2) Ion etching is a very powerful method, but it can produce very small amounts of radiation damage during etching of silicon.

We have solved the problem of pitting during $\mathrm{Si}$ [111] chemical etching.

At the end the best solute is:

$\mathrm{HF}: \mathrm{HNO}_{3}$ : $\left(\mathrm{CH}_{3} \mathrm{COOH}+\mathrm{I}_{2} \mathrm{cryst}\right.$. $)$

$3: 9: 8$

$2.5 \mathrm{~g}$ crystalline $\mathrm{I}_{2}$ per $1100 \mathrm{~mL} \mathrm{CH}_{3} \mathrm{COOH}$

(It is a good idea to dissolve the iodine in a hot acid)

Average etching rate of virgin solute at room temperature is less than 3 micrometers/minute from one side.

Kirill E. Prikhodko

Russian Research Center "Kurchatov Institute", Moscow

\section{Concerning Cleaning the Wehnelt:}

We have been using the ultrasonic bath with "Micro" cleaning solution (Catalog \#6731, International Products Corp. 609-386-5770) to clean the wehnelt, apertures, etc.

I used to use the metal polish and much scrubbing with cotton q-tips, followed by ultrasonic rinsing with $100 \%$ isopropanol. It was always difficult to remove deposits from cracks, and it was not easy to scrub out the tungsten from the small hole in the grid cap.

The solution works best when warm, and I must confess that I use a stronger mix than the instructions dictate (instructions say $2 \%$, I use about $10 \%$ or so in distilled water). I put this brew into a big glass beaker, heat it up on the hot plate (to about $50^{\circ}$ to $60^{\circ} \mathrm{C}$ ), put in the parts, and immerse in the ultrasonic bath. The bath should also have warm water to keep the cleaning solution from cooling off, but this is not imperative. The bath water should also not be too deep, so as to transmit as much energy to the beaker as possible. Also, don't put in parts that can rattle around and scratch each other, but rather clean these separately.

This ultrasonic cleaning solution does a great job, and removes tungsten deposits that I could not previously remove. I became a believer when I observed a fairly large amount of debris collecting in the bottom of the beaker.

The pieces are sonicated in the cleaning solution for at least 10 to 15 minutes, but we have used times as long as 30 to 45 minutes. I use some large tweezers to lift out a piece for inspection and remove it if it looks clean: if not, it goes back in for further sonication.

The cleaned pieces are then sonicated in distilled water for about 2 to 5 minutes, then finished by sonication in $100 \%$ isopropanol for a similar time. This step simply removes all cleaning solution from the parts with distilled water, then removes the water from the parts using isopropanol. The cleaned pieces are then allowed to dry by evaporation.

We just did a column routine on our JEOL 733 using the solution and it also removed deposits from little crevices that were not cleaned that well before.

I have not observed any detrimental effects on the pieces we have cleaned, and am especially interested in hearing about it if it does happen. One should not use this method to clean aluminum parts as it will oxidize the aluminum and produce a black surface deposit.

The solution smells of ammonia, but the ingredients show more than that. I think I paid about $\$ 10$ for 1 quart. Highly recommended.

Paul Carpenter. California Institute of Technology 